Vol. 01, No. 04, octubre - diciembre 2021, 18-29

\title{
Desarrollo de una nueva variedad de frijol arbustivo, clase comercial poroto, para expandido denominado INIA-Huayna
}

\section{Development of a new variety of bush bean, commercial class poroto, for expanded called INIA-Huayna}

\author{
Mirihan Gamarra*
}

\begin{abstract}
* Instituto Nacional de Innovación Agraria, Cusco, Perú.
M.Sc., $\bigotimes_{\text {mgamarra@inia.gob.pe, (D) https://orcid.org/0000-0001-8728-8526 }}$
\end{abstract}

Tel. +51992753260

http://dx.doi.org/10.25127/riagrop.20214.718

http://revistas.untrm.edu.pe/index.php/RIAGROP

revista.riagrop@untrm.edu.pe

Recepción: 27 de julio 2021

Aprobación: 20 de agosto 2021

Este trabajo tiene licencia de Creative Commons. Attribution-NonCommercial-ShareAlike $\quad 4.0$ International Public License - CC-BY-NC-SA 4.0

\section{Resumen}

Se estableció un ensayo de distinción homogeneidad y estabilidad (DHE) en el 2016 para el desarrollo de la nueva variedad INIA-HUAYNA. El diseño experimental fue en Bloques Completamente Randomizados. Los materiales biológicos utilizados fueron INIA-HUAYNA, variedad Qósqo Poroto INIA y ecotipo Chec' che Poroto mejorado. Se evaluó bajo las directivas de la Unión Internacional para la Protección de las Obtenciones Vegetales (UPOV). Los resultados determinaron que la variedad estudiada es distinta en más de 10 caracteres a los frijoles de la misma clase comercial. El hábito de crecimiento es arbustivo con cuatro meses de periodo vegetativo, $87.35 \%$ de índice de expansión, color de Alas Pink violet 20 RHB 75 D, tamaño, longitud y ancho de bractéolas más grandes, intensidad de color verde para hojas, color (RHS149A Dark Green), tamaño, peso de 100 semillas y calibre: 1,75 onza, 59 semillas 1 onza. Los caracteres cuantitativos distinguibles: hábito de crecimiento arbustivo erguido y eficiencia productiva. Los caracteres cuantitativos y pseudocuantitativos son: madurez uniforme, color secundario de vaina, forma de vaina y ápice distintos a los testigos. INIA- 
HUAYNA es única y diferente en esta clase comercial poroto, periodo vegetativo corto y adaptado en zonas productoras de Cusco y Cajamarca.

Palabras claves: Distinción, homogeneidad, estabilidad, poroto, arbustivo.

\begin{abstract}
A Homogeneity and Stability Distinction Test (DHE) was established in 2016 for the development of the new variety INIA-HUAYNA. The experimental design was in Completely Randomized Blocks. The biological materials used were INIA-HUAYNA, variety Qósqo Poroto INIA and improved Chec' che Poroto ecotype; it was evaluated under the directives of the International Union for the Protection of New Varieties of Plants (UPOV). The results determine that the studied variety is different in more than 10 characters to the beans of the same commercial class, where the growth habit is shrubby with four months of vegetative period, $87.35 \%$ expansion index, color of Alas Pink violet 20 RHB 75 D, size, length and width of larger bracteoles, intensity of green color for the leaves, color (RHS149A Dark Green), size, weight of 100 seeds and size: 1.75 ounce, 59 seeds 1 ounce. Distinguishable quantitative characters: upright shrub growth habit, productive efficiency. The quantitative and pseudo-quantitative characters are: uniform maturity, secondary color of the pod, pod shape and apex different from the controls. INIA-HUAYNA is unique and different in this commercial class of beans, short vegetative period and adapted in producing areas of Cusco and Cajamarca.
\end{abstract}

Keywords: Distinction, homogeneity, stability, bean, bushy.

\section{INTRODUCCIÓN}

En el Perú, se cultivan doce especies de leguminosas de grano con más de ochenta clases comerciales, en alrededor de 200000 hectáreas, distribuidas en las 3 regiones y en los 24 departamentos del país, desde el nivel del mar hasta más de 3500 m s. n. m. De las especies cultivadas, los frijoles, pallares y las nuñas, $\mathrm{o}$ porotos, son nativos de Perú y algunos otros países de América Latina (Ministrerio de Agricultura y Riego, 2016). No existe variedades de esta clase comercial, solo se inscribió una variedad en Perú con el nombre de Qósqo Poroto INIA (Gamarra, 2005).

Únicamente en Perú y Bolivia existe aún diversidad en porotos, nuñas o numias. En Bancos de Germoplasma en Instituto Nacional de Innovación Agraria (INIA) en Perú y en el CIAT, las accesiones evidencian la diversidad existente (Voysest, 2000). Parte de esta ha sido estudiada en la Estación Experimental Andenes Cusco, a efectos de desarrollar planes de mejoramiento genético. Desde 1997, se inició trabajos de investigación en Cusco, Perú, a fin de generar una variedad de poroto de hábito arbustivo, de 4 meses de periodo vegetativo. Se han evaluado poblaciones segregantes de poroto de diferentes hábitos de crecimiento generados en Cusco (Gamarra et al., 1996; Castillo, 2006); por hibridación y líneas segregantes procedentes de un vivero remitido por Centro Internacional de Agricultura Tropical (CIAT) en Colombia CIAT, denominado POP (Sevilla, 2009).

Las descripciones de una especie varietal incluyen caracteres distintivos de plantas, que se basan en caracteres cualitativos, cuantitativos, pseudocuantitativo y pseudocualitativos de distinción, uniformidad y estabilidad que permiten distinguir una 
población de plantas distintas homogéneas $\mathrm{y}$ estables que constituirán una variedad (Flores et al., 2011).

Para la liberación de una variedad nueva, se exige realizar la descripción varietal, que permite establecer que la variedad a liberar debe ser distinta, homogénea y estable en relación a las variedades que se encuentran en mercados de semillas (Unión Internacional para la Protección de las especies vegetales, 2004).

Debido a que las variedades, o ecotipos locales, de la clase comercial poroto en Cusco, Cajamarca y Ayacucho tienen periodos vegetativos de 8 a 9 meses y requieren ser sembradas en asociación con el maíz o mediante espalderas porque existe cada vez menos disponibilidad de agua para su cultivo y para favorece su producción, se consideró necesario generar una variedad de poroto de porte arbustivo de 4 meses de periodo vegetativo (Gamarra, 1998; Gamarra et al., 2007; Peralta et al., 2019). Por ellos el objetivo del presente trabajo de investigación fue desarrollar una nueva variedad de frijol arbustivo clase comercial poroto para expandido a ser liberada como INIA-HUAYNA.

\section{MATERIALES Y MÉTODOS}

\subsection{Localización del área de studio}

El Ensayo de Distinción Homogeneidad y Estabilidad se instaló en el Campo Experimental Taray, en el 2016, en Cusco, a una altitud de 2990 m s. n. m. En una latitud: 1325'33" y longitud: 71ํ51'56" (Figura 1).

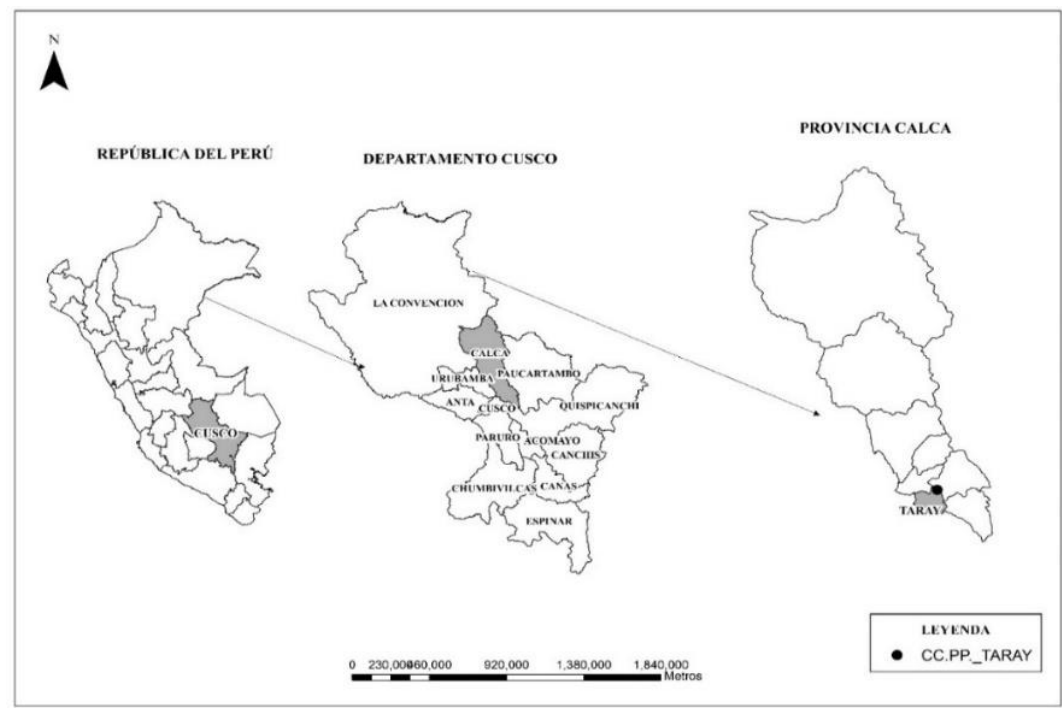

Figura 1. Ubicación del área de estudio.

\subsection{Material biológico utilizado}

Se usó una línea avanzada POP 77 (INIAHUAYNA) de hábito de crecimiento arbustivo. Una variedad de poroto Qósqo Poroto INIA, de hábito de crecimiento voluble (única registrada como variedad, de la misma clase comercial, liberada en 1997) y ecotipo Chec'che Poroto Mejorado, de hábito de crecimiento voluble.
2.3. Directrices para la Ejecución del Examen de la Distinción, de la Homogeneidad y la Estabilidad UPOV TG/12/9 (Unión Internacional para la protección de las obtenciones vegetales, 2015).

Las evaluaciones fueron registradas, en consideración de todos los parámetros determinados para el cultivo de Frijol (Phaseolus 
vulgaris L.), en las directrices para la Ejecución de la Distinción, la Homogeneidad y Estabilidad de la UPOV (Unión Internacional para la protección de las Obtenciones Vegetales Ginebra TG/12/8 y TG/1/3 Introducción General y Conexos TGP).

Se realizaron 16 evaluaciones, en diferentes estados de desarrollo del cultivo de frijol clase comercial poroto y se priorizó: \% de la emergencia de las plantas; crecimiento: caracteres de tallo, hojas, color, tamaño, pubescencia, Floración: Flor, color de ala, color de estandarte, tamaño y forma de bractéolas, pigmentación de estandarte; Fructificación: caracteres referentes a las vainas forma, longitud, ancho, $\mathrm{N}^{\circ}$ de semillas forma. Madurez fisiológica: color del tegumento de la semilla, color del hilium, color primario secundario, presencia de rafe en las vainas o ausencia, longitud y forma de vaina, ápice. Posteriormente a la madurez de cosecha y a la limpieza de granos: calibre, peso de 100 semillas, Índice de Expansión (IE).

Se consideró de acuerdo a categoría de caracteres con asterisco $\left(^{*}\right)$ : todos los incluidos en las Directrices de Examen considerados importantes para la armonización internacional de las descripciones de variedades que deberán ser evaluadas de manera obligatoria en el examen Distinción, Homogeneidad y Estabilidad (DHE) aprobados por la UPOV. Caracteres opcionales (+): los considerados en la tabla de caracteres y las incluidas o priorizadas. Características adicionales: que el investigador considera como características diferenciables importantes entre el cultivar que se pretende registrar y otros cultivares que parecieran ser similares.
Durante el cultivo, solo se efectuó dos controles químicos de masticadores de follaje Diabrotica sp. y deshierbos manuales. A la madurez fisiológica, se procedió a iniciar la cosecha, trilla, venteo y pesaje de rendimientos alcanzados. La cosecha y trilla se efectuaron de forma manual para la posterior selección de granos.

\subsection{Metodología de Evaluación para parámetros cuantitativos, cualitativos $y$ pseudocuantitativos}

Se evaluaron 40 plantas por tratamiento. Se consideraron parámetros cuantitativos, cualitativos, pseudocuantitativos, en flor, planta, hojas, vainas y semillas, según directrices de UPOV y documentos conexos para Frijol o judías (Phaseolus vulgaris L.) TG/12/8.

Se efectuaron evaluaciones por parámetro en cada tratamiento. La diferencia de periodos vegetativos, debido al hábito de crecimiento diferenciado, ocasionó que el trabajo de evaluación sea constante hasta el momento de madurez y cosecha incluso hasta 08-08-2016 en que se evaluó parámetros correspondientes a características de color de grano, forma, calibre, peso de 100 semillas en volubles.

\subsection{Labores culturales}

La densidad de siembra para POP 77 fue 75 $\mathrm{kg} /$ ha y la de los testigos de hábito voluble de 40 $\mathrm{kg} / \mathrm{ha}$ en asociación con maíz. El distanciamiento entre golpes en el poroto arbustivo fue de $0,30 \mathrm{~m}$ y se colocaron 3 semillas, en el poroto voluble intercalado entre golpes de maíz distanciados a 0,50 m. El POP 77:3 semillas golpe, $0,30 \mathrm{~m}$ siembra en monocultivo Qósqo Poroto INIA y Chec'che Local seleccionado, hábito voluble en asociación con maíz Blanco Urubamba, distanciamiento 
entre golpes de maíz $0.40 \mathrm{~m}$, frijol entre golpes de maíz siembra simultánea.

La fertilización química fue 40-60-60 Kg/ha de N - $\mathrm{P}_{2} \mathrm{O}_{5}-\mathrm{K}_{2} \mathrm{O}$. La preparación de terreno $\mathrm{y}$ surcado con tractor, siembra, fertilización, tapado, hasta la cosecha se realizó en forma manual. La cosecha de porotos arbustivos fue el 05 de abril de 2016 y de los volubles fue el 10 de junio de 2016.

\subsection{Diseño y análisis estadístico}

La comparación se efectuó en forma simultánea con frijoles poroto o ñuña de tostar y se consideró la diferencia en periodo vegetativo entre 4 y 9 meses entre hábitos arbustivos y volubles. Otro factor diferenciado fue el sistema de siembra en unicultivo o en asociación con maíz o en espaldera.

Se empleó el diseño experimental Block Completo Randomizados (BCR), con tres tratamientos de 3 repeticiones. La modalidad de siembra para el poroto arbustivo fue en monocultivo y para los testigos volubles en asociación con maíz. Los resultados obtenidos se analizaron mediante el software libre SAS 9,1 .

\subsection{Pruebas de Índice de Expansión o popeado de granos}

Se calculó mediante un protocolo desarrollado en el 2007, en el INIA, en la Estación Experimental Agraria Andenes Campo Experimental Taray. Este ayudó en el proceso de evaluación para seleccionar por este carácter principal de la clase comercial de frijol. Este índice se evaluó con el apoyo de agricultoras y/o transformadoras locales en Cusco y Cajamarca mediante dos métodos: tostado artesanal con Carbonato de Calcio o frito en aceite (Gamarra, 1996; Gamarra 2016).

\section{RESULTADOS Y DISCUSIÓN}

Los resultados de la evaluación de la distinción entre la variedad propuesta INIA-HUAYNA de hábito de crecimiento arbustivo y la única variedad de esta clase comercial, liberada en 1997 en la Estación Experimental Agraria Andenes Cusco del INIA denominada Qósqo Poroto INIA o INIA Frijol Inka de hábito voluble (Gamarra, 1996) y una línea mejorada seleccionada de una colección local conocido como Chec'che Poroto ( Gamarra, 2016), presenta más de 10 caracteres distintivos de un total de 30 caracteres (tabla 1), de los cuales son notoriamente distinguibles 4 caracteres cualitativos (QL), 17 cuantitativos (QN) y 9 pseudocuantitativos (PQ) considerados en el descriptor de la Unión Internacional para la Protección de las especies vegetales, Descriptor de Frijoles o judías (Unión Internacional para la Protección de las especies vegetales, 2004) y, adicionalmente, los relacionados a la clase comercial de porotos que tienen el carácter de popear o expandirse "pop beans" (Ministerio de Agricultura y Riego, 2016). Perú y Bolivia son los únicos países en que aún se siembra esta clase comercial de frijol de tostar. En Carhuaz y Chiquián, Ancash, se hizo pruebas de tostado, sin embargo, no se explicita ni valida protocolos, pero se determinó que la variedad Qósqo Poroto INIA es la que expandía casi en su totalidad (Marmolejo, 2018). Este carácter además podría facilitar a las empresas industriales procesarlas como snacks de alto valor proteico en la alimentación. 
Tabla 1. Resultados y descripción de tipos de caracteres evaluados según Descriptor (Unión Internacional para la Protección de las especies vegetales, 2004)

\begin{tabular}{|c|c|c|}
\hline Carácter & Parámetro & INIA-Huayna \\
\hline $1 \quad$ QL & Planta: Pigmentación antociánica del hipocótilo & Ausente1(RHS144A) \\
\hline QN & $\begin{array}{l}\text { Planta: Intensidad de la pigmentación antociánica del } \\
\text { hipocótilo }\end{array}$ & Débil (3) \\
\hline (*) $\mathbf{Q L}$ & Planta: Tipo de crecimiento & (Ia) mata baja \\
\hline PQ & Solo variedades de mata baja: Planta tipo & No rastrero \\
\hline $6 \mathrm{QN}$ & $\begin{array}{l}\text { Solo de variedades de mata baja: Planta altura } \\
(\mathrm{m})\end{array}$ & $0.44 \mathrm{~m}$ \\
\hline$(+) \mathrm{QN}$ & $\begin{array}{l}\text { Sólo de variedades de enrame: Planta: velocidad a la que } \\
\text { trepa }\end{array}$ & 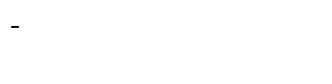 \\
\hline (*) QN (a) & Hoja: Intensidad de color verde & $\begin{array}{l}\text { Oscuro } \\
\text { RHS137ADARK } \\
\text { GREEN }\end{array}$ \\
\hline QN (a) & Hoja: rugosidad & Fuerte (7) \\
\hline $12(+) P Q(a)$ & Foliolo terminal: Forma & Circular Rómbica (7) \\
\hline $13 \quad(+) Q N(a)$ & 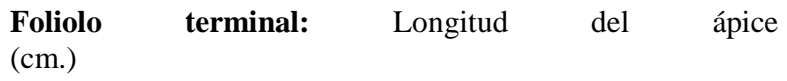 & Medio (2) \\
\hline QN & $\begin{array}{l}\text { Solo variedades de mata baja. Inflorescencias: Ubicación } \\
\text { (en plena floración) }\end{array}$ & Intermedio (2) \\
\hline $16 \quad(*)$ PQ & Flor: Color de estandarte & Rosa (3) RHS70C Pink \\
\hline (*) PQ & Flor: Color de ala & $\begin{array}{l}\text { Violeta (4) Dark Pink } \\
\text { Violet }\end{array}$ \\
\hline $18(+) Q N(b)$ & $\begin{array}{l}\text { Solo variedades de mata baja: Vainas longitud (excluida el } \\
\text { pico) } \\
(\mathrm{cm})\end{array}$ & Corta $3(7.79 \mathrm{~cm})$ \\
\hline $19 \quad(*) Q N(b)$ & $\begin{array}{l}\text { Solo variedades de enrame: Vainas longitud (excluida el } \\
\text { pico) como el } 18\end{array}$ & - \\
\hline $20 \quad(+) Q N(b)$ & Vaina: Anchura & \\
\hline $21(+) Q N(b)$ & Vaina: Espesor & Gruesa $7(1.23)$ \\
\hline $\begin{array}{l}22(+)(*) \mathbf{P Q}(\mathbf{b}) \\
23 \quad(+) \mathbf{Q N}(\mathbf{b})\end{array}$ & $\begin{array}{l}\text { Vaina: Forma en sección transversal (a nivel de una semilla) } \\
\text { Vaina: Relación espesor/anchura }\end{array}$ & Circular 4 \\
\hline $24 \quad(+)(*) \mathbf{Q N}(b)$ & Vaina: Color de base & $\begin{array}{l}\text { Amarillo } 1(\mathrm{RHS} 2 \mathrm{Cight} \\
\text { yellow Green) }\end{array}$ \\
\hline$(+) \mathbf{Q N}(\mathbf{b})$ & Vaina: Intensidad del color de base & $5 \quad$ Medio \\
\hline$(+) \mathbf{P Q}(\mathbf{c})$ & Vaina: Color secundario & $\begin{array}{l}\text { 9(RHS77B Dark Pink } \\
\text { Violet }\end{array}$ \\
\hline $31 \quad(+) P Q(b)$ & Vaina: Forma de la curvatura & \\
\hline $32 \quad(+) P Q \quad(b)$ & Vaina: Forma de la parte distal (excluido el pico) & \\
\hline $33 \quad(*) Q N \quad$ (b) & 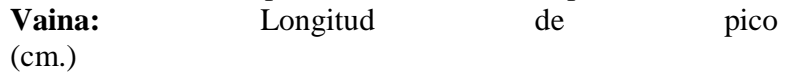 & \\
\hline 35 QN ( b) & Vaina: Textura de la superficie & \\
\hline $37 \quad(*)(+) Q N(d)$ & $\begin{array}{l}\text { Semilla: } \\
\text { (oz.) }\end{array}$ & $\begin{array}{l}5 \text { medio }(1.75 \text { onza }=100 \\
\text { semillas) } 1 \text { onza } 59 \\
\text { semillas }\end{array}$ \\
\hline $38(+) P Q$ & Semilla: Forma en la sección longitudinal & \\
\hline $40 \quad(+) P Q(d)$ & Semilla: Forma en la sección transversal & $\begin{array}{l}\text { Elíptica media } \\
(0.76 \mathrm{~cm})\end{array}$ \\
\hline $\begin{array}{lll}41 & (+) Q N & (d) \\
42 & (+) Q N & (d) \\
43 & (+) Q N & (d)\end{array}$ & $\begin{array}{l}\text { Semilla: Anchura en la sección transversal } \\
\text { Semilla: Longitud } \\
\text { Semilla: Número de colores }\end{array}$ & $\begin{array}{l}\text { Corta } 3(1.23) \\
2\end{array}$ \\
\hline $44(*) P Q \quad(d)$ & Semilla: Color principal (superficie mayor) & $\begin{array}{l}13 \text { Violeta8(Pink Violet } \\
20 \text { RHSN77B }\end{array}$ \\
\hline $45 \quad(*) P Q \quad(d)$ & Semilla: Color secundario & Beige (3) \\
\hline $46 \quad(+) \mathrm{QL}$ & Semilla: Distribución del color secundario & En toda la semilla (3) \\
\hline 48 (*) QN & $\begin{array}{l}\text { Época de floración }(50 \% \text { de las plantas con al menos una } \\
\text { flor) }\end{array}$ & Muy temprana (1) \\
\hline
\end{tabular}

$\overline{\text { RHS MINI COLOUR CHART ([8] Unión Internacional para la Protección de las especies vegetales, 2004) }}$ 
Los parámetros distinguibles fueron $6 \mathrm{QN}, 9(+)$ QN, 10 QN,13(+) QN,14 QN,18 (+) QN,48(+) QN,15 VG QN.

Flor color, tamaño de brácteas de la flor y forma, tamaño de trifolios, intensidad de color y pubescencia en hojas en comparación con testigos de la clase comercial pop beans: Ver figura 2 y 3.

\subsection{Parámetro $6 \mathrm{QN}$ solo variedades de mata baja: Altura de planta promedio}

La variedad propuesta INIA-HUAYNA presenta un nivel de expresión promedio de $0.44 \mathrm{~m}$, con desviación estándar de $0.01 \mathrm{y}$ coeficiente de variabilidad de 2.27 debajo del coeficiente de variabilidad permitido para especies autógamas del $3 \%$, es decir, es homogéneo en el carácter evaluado. En el caso de la variedades testigo de hábito de crecimiento voluble, el descriptor no considera este parámetro para variedades de enrame o volubles por tanto de por si por tener hábito de crecimiento arbustivo es distinta a todas las variedades de hábito voluble.

\subsection{Parámetro $20(+) \mathrm{QN}($ b) Vaina anchura}

La variedad propuesta INIA-HUAYNA presenta un nivel de expresión con promedio de $1.73 \mathrm{~cm}$, con desviación estándar de $0.06 \mathrm{y}$ coeficiente de variabilidad de 3.0 dentro del límite del coeficiente de variabilidad permitido para especies autógamas del $3 \%$, es decir, es homogéneo en el carácter evaluado. Este parámetro en el caso de las variedades testigo
Qósqo poroto INIA y Chec'che poroto de hábito voluble y de madurez heterogénea en la misma planta fue la evaluación al azar ocasionan que la desviación estándar esté entre 0.04 y 0.14, que da origen a coeficientes de variabilidad entre 2.76 a 9.75 plantas evaluadas (tabla 2). Es decir, no se ha observado ninguna planta atípica en Taray y tampoco en las demás localidades donde se le evaluó en Cusco y en Cajamarca por lo cual se considera que la variedad de poroto INIA-HUAYNA es estable.

\subsection{Homogeneidad del cultivo poroto arbustivo INIA-HUAYNA}

Según la evaluación de caracteres cualitativos descritos en la tabla 1, el cultivo de poroto arbustivo INIA-HUAYNA cumple con los requisitos de homogeneidad según la UPOV (Unión Internacional para la Protección de las especies vegetales, 2004)

Los hábitos de crecimiento y la eficiencia productiva se pueden observar en la figura 4 . Con respecto a la diferencia de hábito de crecimiento, con testigos Qósqo Poroto INIA, Chec' che Poroto, en la figura 3c, se observa a la izquierda testigos de la misma clase comercial de hábito voluble en asociación con maíz, a la izquierda y la línea promisoria POP 77 de porte arbustivo a la derecha, con periodos vegetativos en testigos (6 a 8 meses) y POP 77 (4 meses) hábito arbustivo, eficiencia en productividad, precocidad y sanidad. Las características presentadas en las semillas y vaina de INIAHUAYNA se pueden evidenciar en las figuras 5 y 6. 


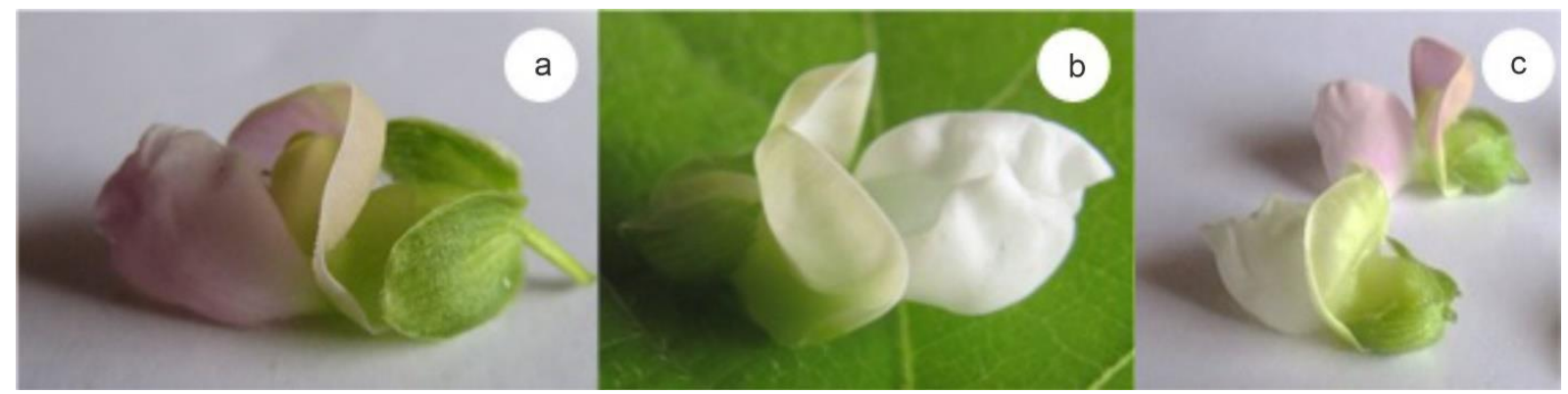

Figura 2. Parámetro 17 PQ: COLOR ALA 4 (Pink violet 20 RHB 75 D) en INIA-HUAYNA (a), 4 (Dark Pink Violet) en Qósqo poroto INIA (b), y 1 (White 60 RHSN999D) (RHS MINI COLOUR CHART, UPOV-2004) en Chec' che Poroto Mejorado (c). En flor INIA-HUAYNA (a), tamaño brácteas: grande 7 (1.02cm), ancho brácteas grandes $3(0.95 \mathrm{~cm})$.

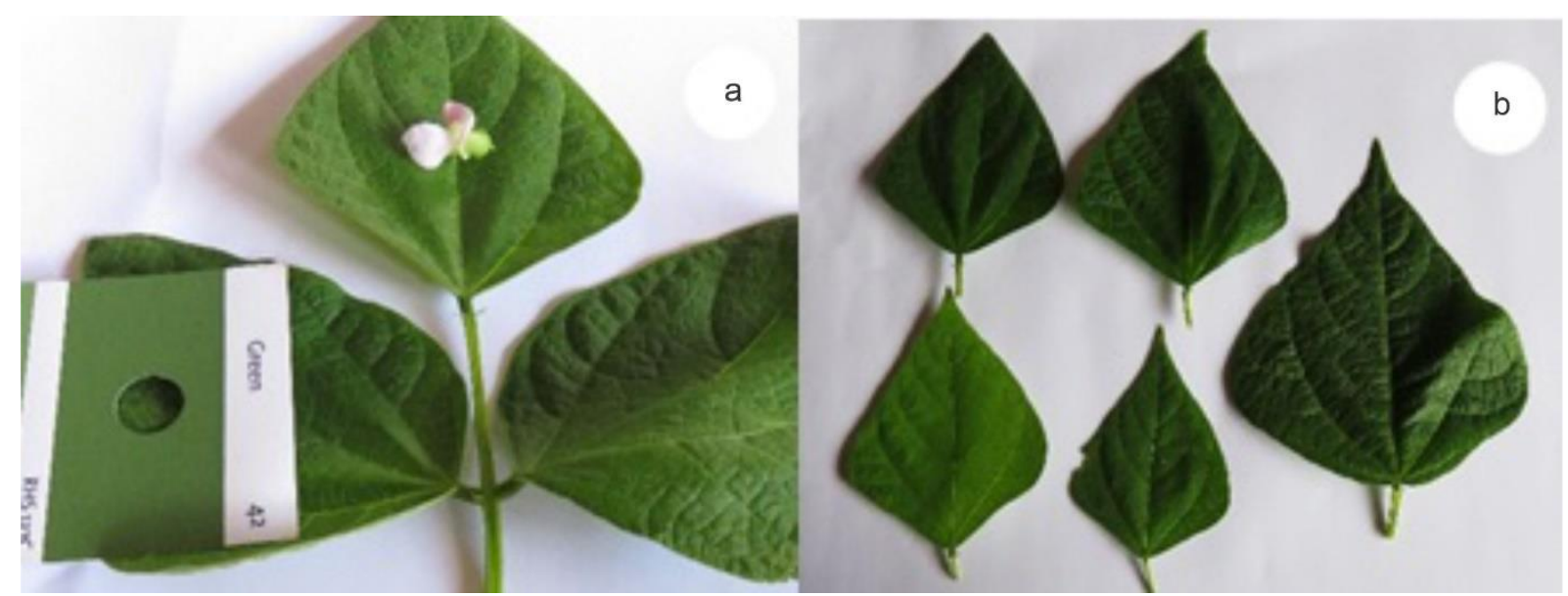

Figura 3. Parámetros distinguibles en hoja, forma, tamaño de trifolios, (a) Tamaño de trifolio Intensidad, Color: 7, Color (RHS149A Dark Green). (RHS MINI COLOUR CHART, UPOV-2004). (b) Intensidad de color (oscura 7 RHS 137y rugosidad Fuerte 7. Foliolo terminal (rómbica 5). Longitud de ápice Medio (2), intensidad de color y pubescencia en hojas en comparación con testigos de la clase comercial pop beans.

Tabla 2. Parámetro $20(+)$ QN (b) Vaina anchura

\begin{tabular}{lccc}
\hline $\begin{array}{c}\text { VARIEDADES POROTO } \\
\text { Nivel de Expresión }\end{array}$ & $\begin{array}{c}\text { Longitud vainas anchura } \\
\text { Promedio }\end{array}$ & $\begin{array}{c}\text { DESVIACIÓN } \\
\text { STANDARD }\end{array}$ & cv \\
\hline INIA-HUAYNA & 1.73 & 0.06 & 3.0 \\
Qósqo Poroto INIA & 1.41 & 0.14 & 9.75 \\
Chec'che Poroto & 1.58 & 0.04 & 2.76 \\
\hline
\end{tabular}




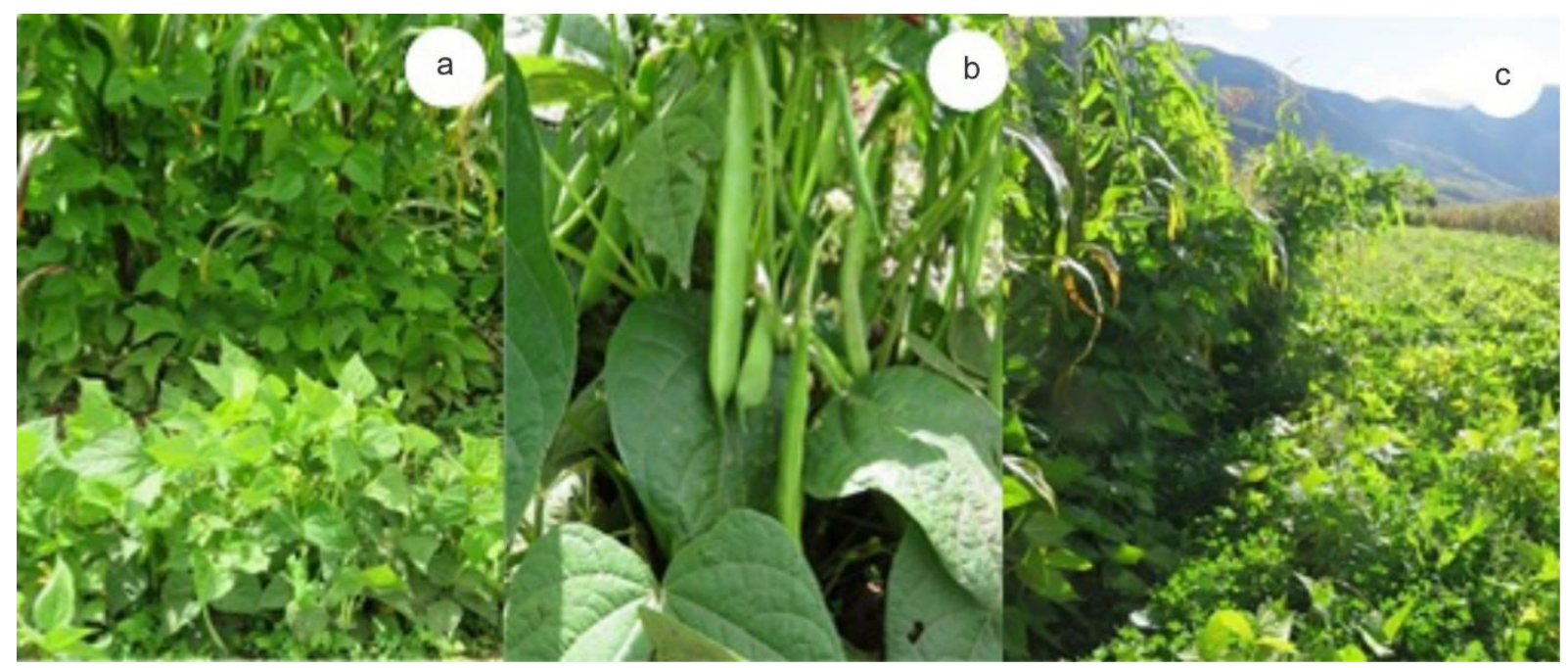

Figura 4. (a) Hábito arbustivo, (b) Eficiencia productiva de la Línea POP 77, (c) Diferencia de hábito de crecimiento con testigos Qósqo Poroto INIA y Chec' che Poroto.

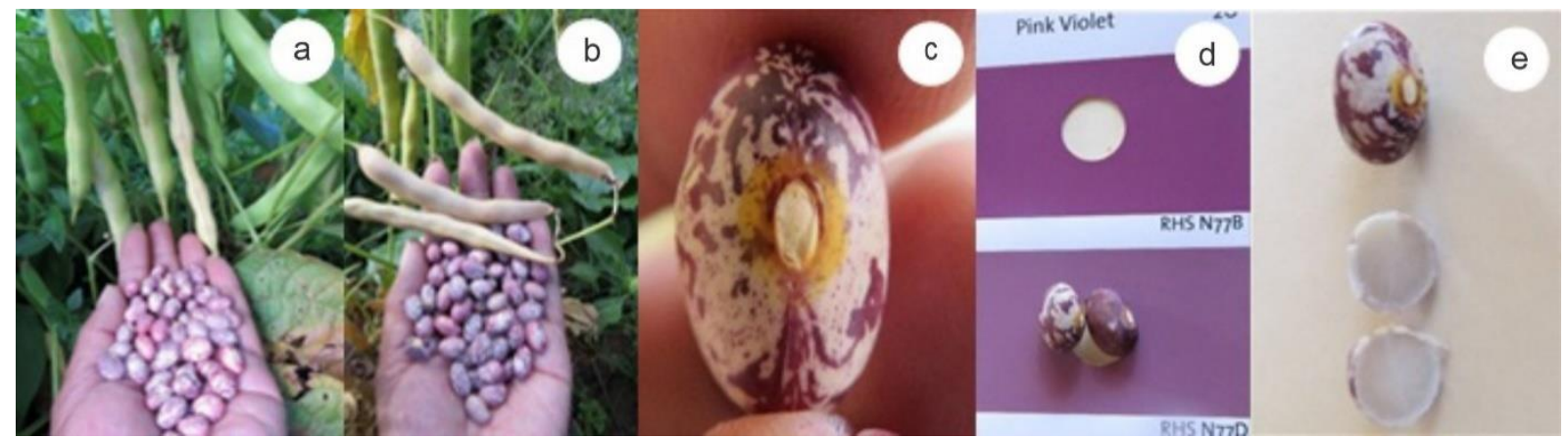

Figura 5: (a) Línea POP 77, Precocidad, forma y color de las vainas, color grano, (b) forma, color de vainas a la madurez, color de grano, longitud de ápice de las vainas, (c) Forma del grano color principal y secundario del tegumento, forma y color de hilium, (d) Color principal de los granos RHS N77D Pink violet, (e) Corte transversal del grano forma ([8] Unión Internacional para la Protección de las especies vegetales, 2004).

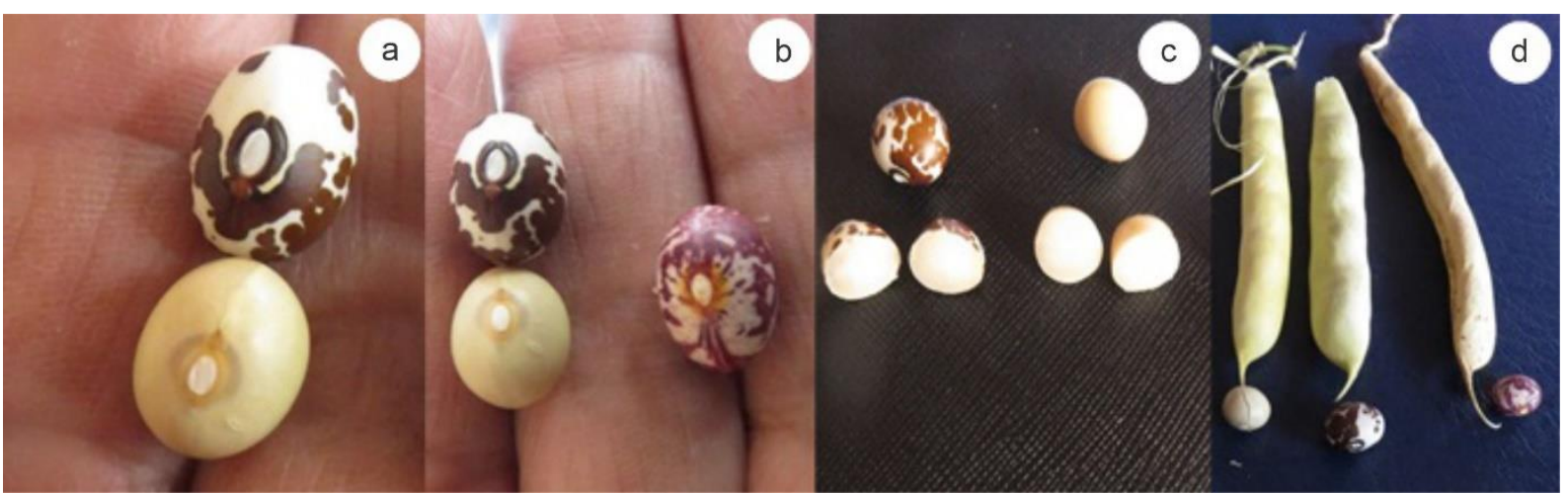

Figura 6. (a) Diferencias notorias de forma, color tamaño granos, (b) Calibre de granos, (c) Distinción en la forma del corte transversal del grano de Testigos; (d) Distinción en forma, tamaño ancho y color de vainas maduras; longitud de ápice de vaina entre POP 77 y testigos Qósqo Poroto INIA y Chec'che Poroto. 


\subsection{Evaluación de la estabilidad}

El cultivo de poroto arbustivo INIA-HUAYNA, en la evaluación de caracteres cualitativos ha presentado estabilidad en las 40 plantas evaluadas, es decir, no se han observado ninguna plata atípica en Taray y se la considera como una variedad estable.

\section{5. Índice de expansión}

En el presente estudio, se encontró que el Índice de expansión (IE) de la nueva variedad de hábito de crecimiento arbustivo es igual a 87.35 \% comparado Chec'che poroto del agricultor con IE de $81.83 \%$ y con la Variedad Qósqo Poroto INIA que tuvo un IE de $86.21 \%$ y con la Selección en Chec'che Poroto, donde se evaluó un IE de $86.19 \%$. La tabla 3 y figura 7 muestra el análisis del índice de Expansión en comparación con los testigos.

Tabla 3. Análisis de Índice de Expansión de granos de poroto de Tratamientos incluidos en estudio DHE

\begin{tabular}{|c|c|c|c|c|c|c|c|}
\hline \multirow[b]{3}{*}{$\begin{array}{c}\text { Peso muestra } \\
\text { Expandido } \\
\text { total }(\mathrm{g})\end{array}$} & \multicolumn{5}{|c|}{$\begin{array}{l}\text { Datos de peso expresados en porcentaje de muestras (g) por } \\
\text { grado }\end{array}$} & \multirow{3}{*}{$\mathbf{I E}$} & \multirow{3}{*}{$\begin{array}{c}\text { Tratamientos /Campaña } \\
\text { agrícola }\end{array}$} \\
\hline & 9 & 7 & 5 & 3 & 1 & & \\
\hline & $\begin{array}{c}\text { bien } \\
\text { expandido }\end{array}$ & Arrosetado & Partido & $\begin{array}{l}\text { Duro } \\
\text { resquebrajado }\end{array}$ & $\begin{array}{l}\text { Duro no } \\
\text { expande }\end{array}$ & & \\
\hline 487.9 & 66.10 & 13.51 & 4.50 & 4.28 & 11.60 & 81.83 & $\begin{array}{l}\text { Chec'che poroto (testigo } \\
\text { del Tostador } 14-15 \text { ) }\end{array}$ \\
\hline 311.0 & 79.58 & 4.82 & 1.61 & 7.07 & 6.91 & 87.35 & POP 77 14-15) Mollepata \\
\hline 317.0 & 58.39 & 27.52 & 9.17 & 3.50 & 1.42 & 86.21 & Q.P TARAY (15-16) \\
\hline 445.5 & 60.04 & 26.30 & 8.77 & 1.26 & 3.64 & 86.19 & Chec'che poroto (15-16) \\
\hline
\end{tabular}

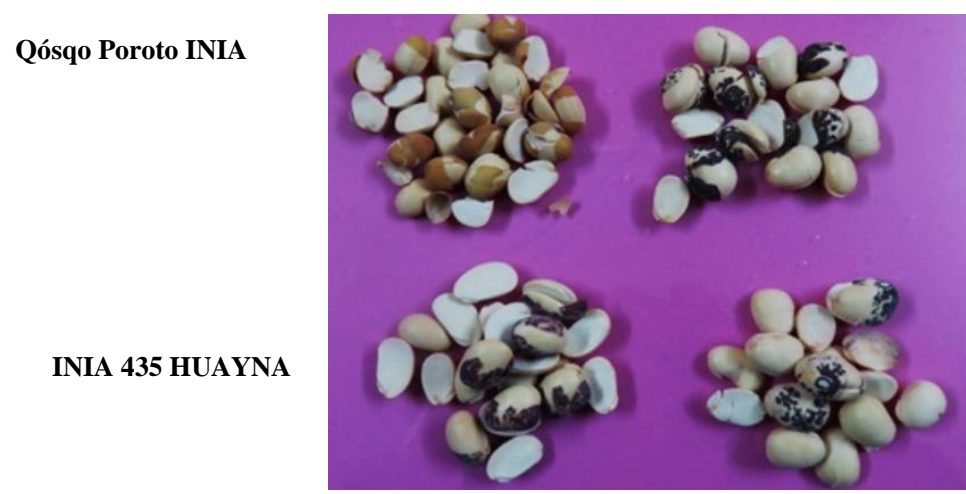

Qósqo Poroto INIA

Figura 7. Índice de expansión de la línea POP 77 y los Testigos utilizados.
Chec'che Poroto tostador testigo

Selección en Chec'che Poroto 


\section{CONCLUSIONES}

La nueva variedad de poroto arbustivo INIAHUAYNA (POP 77) es distinta en más de 10 caracteres a los frijoles volubles de la misma clase comercial y completamente diferente a otras variedades arbustivas de frijol común. Además, presenta caracteres distinguibles como hábito de crecimiento arbustivo de periodo vegetativo de 4 meses, en siembra con arquitectura erguida de la planta, sanidad y eficiencia productiva en contraste con testigos volubles.

Esta nueva variedad de poroto tiene buen índice de expansión, resistencia a principales enfermedades y amplia adaptación en las principales zonas productoras en Cusco y Cajamarca. Por su hábito de crecimiento, será más fácil promover su cultivo transformación y consumo dado su alto valor nutritivo y las potencialidades de transformación del grano.

El área de Leguminosas del Instituto Nacional de Innovación Agraria INIA-Perú, en base a los resultados contenidos en el Estudio de Distinción, Homogeneidad y Estabilidad refiere el sustento técnico para inscripción y liberación de la línea promisoria POP 77, como una nueva variedad mejorada de POROTO con el nombre de INIA-HUAYNA, resultado del trabajo innovador y participativo entre INIA Estación Experimental Andenes Cusco, Estación Experimental Baños del IncaCajamarca, de agricultores productores de poroto, ñuña o numia en los dos departamentos.

\section{Agradecimientos}

Financiamiento externo a través de los proyectos PROFRIZA (Dr. Mathew Blair),
PREDUZA (Dr. Daniel Danial), KolFACI CIAT; participación de agricultores $y$ trasformadores artesanales poroto expandido: Sra. Julia Chalco y Sr. Emilio Gil Caviedes; en los procesos de investigación y validación técnica y económica, así como el apoyo técnico de Julián Arana Quispe y Jorge U. Flores Tito en Cusco y en Cajamarca de Ing. Agro. Juan Chaupe Cabrera, Ing. Agro. Toribio Norberto Tejada. Colaboraron Ing. Agr. Sergio Cardoso Villacorta e Ing. Agr. Yubriel Herrera en EAE Limatambo en el Marco del Proyecto FrijolConsorcio Andino.

\section{Referencias}

Castillo, L.A. (2006). Evaluación y selección de cuatrocientas treinta y seis lineas de ñuñ o poroto (Phaseolus vulgaris L.) por su capacidad de expandido. [Tesis de grado, Universidad Nacional de San Antonio Abad del Cusco, Perú].

Flores, A., Vásquez, M. E., Borrego, F. \& Sánchez, D. (2011). Análisis de la homogeneidad, distinción y estabilidad de tres variedades sobresalientes de tomate. Revista mexicana de ciencias agrícolas, 2 (1): 516.

Gamarra, F. M. (1998). Evaluación y Selección de Progenitores para Resistencia a Antracnosis en Invernadero. Informe Proyecto PREDUZA, Año 1 INIA DGIA-Cusco -Perú.

Gamarra, F. M. (2005). Avances en el Desarrollo de Variedades de Frijol Clase comercial Nuña o Poroto de habito arbustivo. Articulo INIA.

Gamarra, F. M. (2016). Estación Experimental AndenesCusco Programa Nacional de Investigación en Cultivos Andinos- Qósqo Poroto-INIA, Cusco, Perú. 13pp, 1996.

Gamarra, F. M. (s.f.). Memorias Anuales del Programa Nacional de Investigación en Cultivos Andinos.

Gamarra, F. M., Puma, U. J., Arana, Q., Ortiz, J. y Vidal, A. (1996). Manual Qósqo Poroto-INIA, 13 pp.

Gamarra, F. M., Tejada, C. T. \& Arana, Q. J. (2007). Desarrollo de Variedades de Frijol Nuña o Poroto Arbustivo de habito arbustivo en el Perú. Revista de la E. E. A., vol. 4, no 7, pp. 23 -27pág. 
Marmolejo, K. J. (2018). Variabilidad Genética del frijol común tipo ñuna (Phaseolus vulgaris L.) en las localidades de Carhuaz y Chiquián, Ancash. [Tesis de post grado, Universidad Nacional Agraria la Molina, Lima, Perú].

http://repositorio.lamolina.edu.pe/bitstream/handle/ UNALM/3398/marmolejo-gutarra-karinajessica.pdf?

Ministerio de Agricultura y Riego (MINAGRI) (2016). Leguminosas de Grano "Semillas nutritivas para un futuro sostenible". 1era edición, GALU GRAF S.A.C., Lima, Perú. 75 pp.

Peralta, I. E., Peralta, I. F. \& Peralta, I. H. (2019). Lúdica y Juegos con el Fréjol en Ecuador, Perú y Bolivia. 213 pág. 112-114 p. Editorial: Quito, EC: INIAP, Estación
Experimental Santa Catalina. 216 pp. Estación: E. E. Santa Catalina, 2019.

Sevilla, R. (2009). Mejoramiento de Granos Andinos con potencial para asegurar la Nutrición Popular y el Alivio de la Pobreza. 5 pág.

Unión Internacional para la Protección de las especies vegetales (UPOV). (2004). Descriptor de Frijoles o judías - Ginebra TG/12.8,9 2004 Original Ingles. (Phaseolus vulgaris L.).

Unión Internacional para la protección de las obtenciones vegetales (UPOV) (2015). Directrices para la ejecución del examen de la distinción, la homogeneidad y la estabilidad. 47p.

Voysest, V. O. (1996). Mejoramiento Genético del Frijol CIAT N ${ }^{\circ}$ 321. Colombia, 2000. 